\title{
Collaborative Networks and Technology Clusters - The Case of Nanowire
}

\author{
Sercan Ozcan, Nazrul Islam \\ School of Management and Business, Aberystwyth University \\ Penglais, Aberystwyth, SY23 3DD, United Kingdom \\ E-mail: $\underline{\text { seo5@aber.ac.uk; mni@aber.ac.uk }}$
}

\section{Abstract}

Patenting activities and technology diffusion in high-tech sectors are being increasingly driven by collaborative, international and technology-based new entrants. In the realm of nanotechnology, one of the most mature structures is nanowire. This paper is concerned with the technology transfer process in the nanowire field; in particular it examines how patent collaborations occur and how the key actors interact with each other to support this process. This study uses a different methodology than previous studies in terms of patent data extraction. The methodology offers a new taxonomy that could make a significant impact on accurate patent data quests and increase the reliability of patent analyses in emerging fields such as nanotechnology. As patent data are valuable sources of technology innovation data and for forecasting technical change, this study utilises patent network analysis to visualise the actors' clusters and their relationships at the organisational, national and international levels. Overall, this study proposes a new collaborative network model to assist with analysing patenting activities between actors in regard to types of linkages. Different types of linkages between countries and organisations can be found for nanowire-related patenting activities by following the proposed network model. Findings indicate that some nations have highly centralised networks where large organisations dominate most linkages, as in the case of South Korea with regard to Samsung. Nations such as the US and Japan have a more distributed network where academic and industrial players are linked with each other. In the case of China, there were mono-linkages between large organisations such as Foxconn and Tsinghua University, which was key with regard to collaborative innovation there.

Keywords: nanotechnology, systems of innovation, patent analysis, collaborative networks, clusters, linkages, nanowire 


\section{Introduction}

30 Nanotechnology is the process of understanding, manipulation and production of materials and devices at the level of atomic and molecular precision [1], particularly at dimensions of roughly 1 to 100 nanometers, where unique phenomena enable novel applications. This field is highly interdisciplinary [2-4], as it depends on the knowledge and expertise found in conventional disciplines such as chemistry, physics, biology, material sciences and medicine [5]. For this reason, there is much varied research being conducted in order to gain insights into this field and to forecast its possible outcomes. The wide range of studies in this field may increase the rate of nanotechnology diffusion and shorten the pre-commercialised era, and so help it to move on to its highly commercialised era. However, the outcomes of current nanotechnology innovation systems, with their commercial progress, their possible positive and negative effects on the environment and existing industries (e.g. whether they are disruptive innovations or the extent of their market penetration) are uncertain [1, 6-8].

The uncertainty of nanotechnology in a business context is even higher when the subcategories of this field are considered. Nanomaterials, nanomedicine and nanoelectronics are some of these subcategories of nanotechnology. However, the applications of these subcategories have differences; there are common nanostructures and nanoparticles that are used in these different fields of research such as nanotubes, nanowires and nanocrystals. All these nanostructures have different characteristics and their own particular novelties [9]. For this study, the nanowire field was chosen for analysis, there being two main justifications for this. Firstly, nanotechnology is not a clearly defined sector, so the scope of this study requires specifying linkages between countries or organisations for a specific field. Secondly, the field of nanowires is one of great interest for researchers and industry, when the number of granted/applied patents for this technology is considered. According to the collected patent 
data, 4484 patents out of 49544 nanotechnology patents are for nanowire, which represents almost $10 \%$ of all nanotechnology patents.

Reliable and valid information about a particular technology or innovation system can be gathered if the patent data is analysed systematically $[10,11]$. Some of the reasons why patent analyses are pursued include the discovery of promising technologies, assessment of technological advances and new trends, or helping organisations in their strategic decisionmaking [12]. Patent analysis can benefit various individuals and organisations such as inventors, R\&D departments, policy-makers, academics and managers. Generally, looking at various patent analyses, the most commonly used methods are bibliometric and quantitative analysis; if some of these studies are clustered under various categories, these can then be subjected to network analysis, citation analysis, trend extrapolation/impact analysis, life cycle analysis, innovation system modelling, road mapping studies and economic base analysis [13-17].

Relevant studies conducted by Huang et al. [18] present a longitudinal patent analysis on nanotechnology patents between 1976 and 2002, focusing on content map analysis and citation network analysis. Accordingly, they showed how countries, institutions, and technology fields are linked with each other in terms of cited and citing actors by visualising linkages of the largest patent citation centre, institutional patent citation centres and dominating technologies that are cited most. Another similar study by Li et al. [19] identified key influential players and subfields, knowledge transfer patterns, and overall knowledge transfer efficiency. Porter and Youtie [20] examined nanotechnology positions in relation to other disciplines by considering their multidisciplinary nature, and linkages of these disciplines amongst each other. Similar work was conducted by Miyazaki and Islam [17], focusing on cross-country comparisons, actors and institutions by using similar quantitative 
nanotechnology from a global perspective. Shapira et al [21] observed the influence of crossborder international invention linkages by using patent data. Our study differs from the previous studies, as the focus of this work is to examine the types of linkages by focusing on co-ownership of patent documents rather than citation linkages. Patent co-ownership analysis is a better model for this study since our objective is to study collaboration linkages between actors, while citation analysis is a more appropriate method for studying knowledge flow between actors.

The objective of this paper is to analyse various linkages by examining granted and applied nanowire patents until the present time. To assist with the investigation process of types of linkages within a network, a new collaborative network model is proposed. This model is tried with the nanowire case bearing in mind the international and organisational contexts that assist gathering information on collaboration trends, linkages and the key players. The case of Samsung is analysed to examine a cluster and to support the findings further.

Considering the limited number of studies in this field in terms of collaborations in patenting activities, this study contributes to the field with a specific case of nanowire patent analysis. There are few studies that examine how nano-patents are linked to each other and in what form they are interconnected. In any event, there is a need for up-to-date studies in various areas of nanotechnology, as it is an emerging field undergoing rapid development. In this study, the patent collection method and the search query are well defined and the patent database was the best among those available for use. The accuracy of the patent database was increased by using lexical queries with a combination of patent classification codes. 


\section{Theoretical Background}

101 In the course of time, innovation management theories have evolved and the perspective on

102

103

104 how innovation processes work has changed. After Schumpeter's identification of innovation and his studies [22], there were various theories that have been used by technology or innovation management specialists. The first theories that received attention and were implicit in the work of many innovation specialists were the technology push [23-26] and market pull theories [26-30]. These models were widely accepted in the technology management field but until the 1990s, they failed to take account of other influences that were affecting the innovation process. Lundvall [31] introduced a more comprehensive model to explain systems of innovation. In this model, linkages of various actors were taken into account in the innovation process and included many actors under a single system. Various important aspects are highlighted such as the functions of actors, linkages of actors, and knowledge flow between them. As the focal point of this study is the collaboration mechanisms within an innovation system, the relevant literature is thoroughly reviewed in the following section.

\subsection{Systems of Innovation Approach}

The system of innovation (SI) concept has captured the attention of a growing number of researchers involved in the fundamentals of SI as it explains the system in terms of actors, processes and flow of information. The SI comprises the linkages and flow of information among actors such as inventors and organisations in terms of innovative processes [31-35] and describes the processes of interactions among the actors to facilitate the innovation value chain $[35,36]$. Various SI studies are described in the literature, including national systems of 
innovation [31, 37, 38], regional innovation systems [39, 40], sectoral systems of innovation

124 [41], technological innovation systems [42], and functions in innovation systems [43].

Looking at these different models, the notion common to all of them is to explain how an innovation system develops, diffuses, and utilises innovations within different contexts. However, the focal point of each study varies at some level and these studies emphasise different aspects of innovation systems. For each approach, the innovation system model differs in terms of the concepts used and the actors identified and highlighted.

The regional innovation system model describes the dissemination of knowledge within a geographic area that is at the regional level. By regional, this study means a region within a country such as the London area in the UK, or California in the US. The main characteristic of this model is the fact that it examines collective learning processes among regional actors in a particular technology or industry. It stresses the advantages that are gathered from a localized innovation group and different kinds of innovation cultures, norms and linkages in terms of the way knowledge is created and disseminated between regional actors [44]. The national innovation system has many similarities to the regional innovation system in terms of actors considered and the way they are linked to each other. Sectoral systems of innovation, on the other hand, involve the analysis of innovation processes, the linkages between innovation and industry, the determinants affecting innovation and the international performance of organisations and nations in various sectors [41]. There are three variable groups that are explained in this model: knowledge and technologies, actors and networks, and institutions [41].

Having examined the similarities and differences of various models in SI research, it can be seen that one of the key aspects within an innovation system is the structure of the 
collaboration mechanism among actors. For this purpose, models related to the type of networks, clusters, and linkages of actors are examined in the following section.

\subsection{Collaboration Models and Network Types}

Collaboration is a course of action in which actors share information, resources and responsibilities in the attainment of a common goal that is jointly planned, implemented, and evaluated by the participants [45]. There are different collaboration models including informal collaborations, strategic alliances, joint ventures, partnerships, R\&D consortia, licence agreements, coalitions, associations, clusters and networks. Networks function over linkages between individuals, organisations and shared interests. Sometimes networks can form formal or informal structures within or outside a partnership setting. Basically, networking involves communication and information exchange for mutual benefit. The difference between clusters and networks can be described by four dimensions, which are geographic, industry sector, nature of the relationship, and objectives [46]. Clusters are generally distinct from networks in that the geographical linkages between partners are from a set of associated sectors while such linkages in networks may come from a variety of fields or sectors [47]. Networks of organisations do not have to be limited to a specific geographical area, and a particular sector and its structure of networks can be designed in such a way as to allow active collaboration [46].

Collaboration networks can take different forms, for example that of an industry cluster [48]. Industry clusters are the primary stage and comprise a group of companies which are characteristically located in the same region and form part of a common industry [48]. Due to regional and sectoral bonds, an industrial cluster aims to escalate the overall competitiveness of its members in their region and also tries to expand it to other regions. Some of the 
benefits of being a part of such a collaborative network can be sharing information and expertise such as buyer/supplier externalities, or making use of common resources such as technological tools, or providing support to each other when various business opportunities/challenges arise. Patents can be a part of this kind of collaboration, as sometimes patents are used as barriers. However, patents may also be the starting point of an industry cluster in terms of spin-offs and academic institutions.

Nanotechnology can be classified as a science-based cluster [49] which is highly R\&D-and patent-focused and is likely to have a close relationship with the public research sector (i.e. universities, government research bodies etc.) This is due to their requirement for basic research and so it is essential for the public research sector to become involved for there to be an effective innovation structure.

There are various models that analyse linkages of actors within an SI, for example the triple helix model, the TEN model, and network models. These different models are examined to gain information about the structure or types of linkages within an SI. The Triple Helix concept comprises a model for collaborative relationships between three major institutional spheres that comprise universities, industry and government, in which innovation is an outcome of interaction. This model presents manifold mutual relationships at various stages of the knowledge capitalization process [50]. There are three main different actors within this model and these actors may or may not be linked effectively in terms of patenting activities. Through patent analysis, it may not be possible to see the linkages between government and other actors, as the fund providers cannot be identified through patent analysis. However, it is possible to identify the linkages between academia and industry and relate this information to the model. This model can be used to understand insights of interactions between two spheres, which are academia and industry. 
Another framework that illustrates the roles and linkages of actors within an innovation system is the Techno-Economic Network (TEN) [5]. The TEN framework is a useful framework to analyse the systems of innovation in a comprehensive manner for a chosen sector [5]. The TEN concept is an effective framework when the aim is to study an innovation system at a large scale, to consider its complexity. There are four different poles within the TEN framework and it has been organized around three major poles that are technology, science, and market. Another minor pole that is presented within this system is the Finance Pole, due to its indirect players or innovation links. Each of these poles is categorized by the type of actors and intermediaries in regard to their duties. Intermediaries vary in terms of tangible and intangible resources for those actors within TEN. Moreover, it shows how the poles are linked to each other in terms of their direct or indirect linkages and also it shows which intermediaries they are linked by, for example the Transfer Pole (between the Science and Technology Poles) and Development Pole (between the Technology and Market Poles). Following this model it should be possible to identify various collaboration mechanisms within a system. Even though the TEN model and the triple helix model illustrate actors and their linkages, these models do not identify collaboration mechanisms in regard to types and formation of networks.

Having examined different collaboration methods, the network structures of these linkages should be analysed as well. One can assume there would be academic and industrial linkages in nanowire patenting activities, but it is not clear if the form of linkages consists of small clusters or a network on a larger geographical scale.

One of the basic categorisations of networks describes them as centralised, decentralised, or distributed [51]. Accordingly, there can be a network with a dominant central ego to which other nodes are directly linked. This network may not have a very healthy structure as the network is controlled by an individual organisation and the progress of the network may be 
219 slow and unstable. The structure of a network is likely to be vulnerable and unstable if there

220 is a single node in it, as it is too dependent on the central ego.

221 A decentralised network can be considered as a more efficient model in terms of knowledge

222 flow compared to the centralised model, as the structure consists of clusters or smaller

223 networks with a higher number of central organisations. The most effective and stable

224 network structure is the distributed network, as risk factors are lower compared to other types

225 of networks. Distributed networks are likely to have lower levels of formalised interactions

226 among comparatively equal organisations and the distribution of knowledge and resources

227 will be more balanced.

228 Considering previous models, the following collaboration model in Figure 1 is being proposed as an analytical framework for this study. The core idea of this paper is to analyse the network structure and collaboration system of this particular field. However, it is assumed that there will be various structures where there are central players or multiple dominant actors appearing within nanowire SI networks. The proposed model consists of five different network linkage types, which for the purposes of this study have been termed: mono-linkage, oligo-linkage, central-linkage, decentral-linkage and distributed linkage (see Figure 1). Considering the triple helix and TEN models, it is expected that there will be various types of linkages in terms of actors and information flow. For example, a mono-linkage might exist between an academic and an industrial player where the information flow is between the science and the market poles.

This study will apply the proposed model to analyse the nanowire case, where the institutional networks of nanowire technology will be examined in terms of the structure by which organisations are linked to each other and what the national differences are with regard 
243 to the various network types as previously described (i.e mono-linkage, oligo-linkage, 244 central-linkage, decentral-linkage and distributed linkage), and what the network

245 characteristics are for nanowire technology. To fulfil the purpose of the study, it attempts to 246 answer the following fundamental questions: 1) how the leading actors are linked to each 247 other and how effective their network is; 2) what the collaboration trends are in respect to the 248 dominant and emerging actors in the nanowire case; and 3) what the current network structures are in terms of the linkages between organisations.

\section{Methodology}

252 The present study applies tech-mining methodology, proposed by Porter and Cunningham

253 [52], combining bibliometrics using patent abstracts from patent databases. Tech mining analyses relations between actors and technologies within a given innovation system, using specialist keywords, derived from the Nano Science and Technology Institute publications. The subsequent analysis was performed using dedicated tech mining software Thomson Data Analyser (TDA), automating mining and clustering of terms occurring in article abstracts and article descriptors such as authors, affiliations or keywords. The outline of this paper, including methodology and the general process, can be seen in Figure 2. In general, gathering the valid patent data, efficient analysis of large data sets, and handling and interpreting the outcomes of the analysis is crucial for the accuracy of the results. There are crucial steps for tech mining analysis and these are: searching for required data (e.g., key terms), gathering the required data (patents or publications), importing data into text mining software (e.g., Thomson Data Analyzer, VantagePoint), cleaning and optimization, and analysis and interpretation of results. These steps are explained in the methodology section. 
266 In the methodology section, sampling and its link to generalizability and quality of 267 implications is key to the whole research process [53]. It is essential to justify the type of

268

269 samples for the internal and external validity of this research [54]. The type of sampling, and the external and internal validity of results are highly interconnected, as will be explained in the following section.

[Insert Figure 2 here]

\subsection{Taxonomy of Patent Databases}

In considering the validity and reliability of this research, one of the key issues is to use an expedient patent database in terms of the required size and the coverage of patents. For this purpose, various patent databases were compared to find the best offering in terms of the number of patents offered and the coverage of patent authorities as shown in Table 1 . The strengths and weaknesses of each patent database are considered.

Delphion is a reasonable tool for quick or occasional patent searches; however, it is not ideal for detailed patent analysis compared to other systems, as there is a ceiling of 500 patent documents. MicroPatent has more advantages compared to the Delphion patent database given its 20,000 hit list and 20,000 patent documents export option; however, if the research area is about a broad and mature field, MicroPatent is likely to be insufficient as the required data would be larger than 20,000. Moreover, the data coverage of MicroPatent is smaller than its competitors. PatBase offers the highest number of patent authority coverage and the greatest hit list of 100,000 . However, the export option is limited to 20,000 records per month and this would be a drawback if the required patent database is higher than 20,000, giving it the same drawback as MicroPatent. Thomson Innovation has a significant number of patent authority coverage but it is smaller than Patbase's coverage. The maximum offered hit list is 
60,000 which is lower than the PatBase offering. The total export option is 60,000 with an analyst subscription, which gives it the highest export option compared to its competitors. Also, it is possible to download the maximum allowed records more than once, so it is possible to gather the patent documents even if there are more than 60,000 records by breaking them down to the required level by year or by sub-category.

For this research, some criteria were crucial, namely the patent authority coverage, maximum hit list, availability of various patent database export options and the maximum allowed export quantity of patent documents. This is due to the fact that the required patent database was large and exceeded some of the patent database providers' maximum allowed patents document export options. Delphion and MicroPatent provide a limited number of patent authorities while their competitor, PatBase, does have a significant degree of patent authority coverage but there are service restrictions in terms of search hit list and the number of patent documents that would limit the potential data size. As a result of this comparison between various patent database providers, Thomson Innovation was the preferred patent database as the required large data set could be gathered and analysed by TDA. Additionally, the provider of the Thomson Data Analyser and Thomson Innovation patent database is the same organisation so the patent data and the software are optimized in the TDA export function and therefore the gathered results are improved even further.

\section{[Insert Table 1 here]}

\subsection{Patent Data Collection Method}

One of the biggest challenges in a patent analysis is to gather required patent data by selecting the appropriate terms for the search so that the data set includes the relevant patents and excludes unnecessary patents, thus increasing the validity of the research. Moreover, it is 
an even greater challenge if the analysed field is an emerging technology and there are many similar terms that are used by other technologies. In the case of nanotechnology, the USPTO created a nanotechnology patent class labelled 977 in 2005 as a cross-reference art collection, and its sub-categories, to gather all the nanotechnology related patents within this category. Class 977 presents additional collections for patent searches, but it is not very useful for categorizing patents as a basis for assigning applications. Nanotechnology related US patents are only classified in class 977 as a secondary or a cross-reference classification and they are not primary classifications. For primary classifications, B82 by IPC is used and this classification is very helpful if nanotechnology patents are required to be analysed in terms of nanotechnology's sub-domains or sector analysis. This was a great approach considering the consistency of the nanotechnology related patent analysis, as this field is very dispersed among various fields such as electronic biological and robotic applications. The negative aspect of this new nanotechnology patent classification is that nano-related inventions were patented first in the $80 \mathrm{~s}$, so many patent authorities such as USPTO assigned teams to reclassify the records of patents granted previously to the established nanotechnology patent many nanotechnology related patents had been introduced with different patent classifications. However, the majority of existing nanotechnology related patents have been reclassified into their respective patent classifications and new nanotechnology patents are classified into the required classification. The main problem in finding nanotechnologyrelated patents is that there are some patents within the nanotechnology class that are not related to the nanotechnology field (e.g. the following patents have been classified under the patent code B82; however, they are not really at the nano level, please see the patent documents: WO2001097295 A3, EP1688735 B1 and WO2012047042 A3). 
338 Various approaches are followed by patent analysts and researchers in this field. There are many limitations and drawbacks in terms of the search terms that are used and the nanotechnology patents which are obtained. There are two main approaches in this field. One of the approaches is to use all the required nanotechnology related terms such as nanotube, nanowire and nanosensors in the patent search and to try to get the highest possible hit list as a result. This type of search may face two major problems. The first one is that the researcher may not cover all the required nano-terms and as a result may not be able to access all the required nanotechnology related patents, for example colloidal crystals, quantum dot, and fullerene do not include the term "nano", but they involve nanotechnology-related patents.

Another issue with this type of research is that there are many patents that mention nanotechnology-related materials within patent documents that are not for a nanotechnology invention. For example, if the details of some of the patents are analysed, it can be seen that the nanotechnology-related term is used in the description of a non-nanotechnology patent that states the invention can also be used with one type of nanomaterial such as nanotube. As a result, it is possible to include unnecessary patents and exclude necessary patents in the analysed patent data set.

The second common approach in nanotechnology-related patent analysis is to obtain all the patents that include terms that start with prefixes as "nano" or "quantum" by using Boolean search logic such as nano* OR quantum* and excluding all the unnecessary patents from the result which include terms such as nanosecond and nanometre. The problem with this approach is that there are many nanotechnology-related patents that include those terms, for instance there are many nanotechnology patents that include both 'nanowire' and 'nanosecond'. Also, as was explained with the previous approach, there is a possibility of obtaining unrelated patents that mention the possible compatibility of a particular nanomaterial or nanoparticle with the patented invention. 
363 Given the limitations and drawbacks of the above approaches, it was thought that the best nanotechnology search practice would be to use all available nanotechnology classifications to gather all the nanotechnology classified patents such as 977 by USPTO, B82 by IPC, Y01N by ECLA and 3C082 by Japanese F-Terms. All irrelevant patents classified within these categories could be eliminated by using Boolean search logic with very broad nanotechnology related terms, such as 'nano*', 'quantum*' and 'fullerene*'. Afterwards, the DWPI (Derwent Patent Index) is used to exclude patents that appeared more than once in the search results, as, due to nature of patent applications, inventions are granted more than once in various patent authorities to secure the invention in that respective country or region. For the nanowire case, the following search terms are used;

$(\mathrm{AIOE}=(\mathrm{B} 82 *) \mathrm{OR} \mathrm{FIC}=(\mathrm{B} 82 *) \mathrm{OR} \mathrm{UCC}=(977 *)) \mathrm{AND}\left(\mathrm{ALLD}=\left(\right.\right.$ nanowire* ${ }^{*}$ or nano-wire* or quantum ADJ wire* or nano ADJ wire*))

Establishing the validity and reliability of the collected patents in the nanotechnology field is a great challenge. To explain how the collected data differs from the existing studies, four different "nano"-related patent categories are introduced. The first of them comprises relevant nanowire-related patents. The second type of patents includes nanotechnology-classified patents with nanowire-related terms but which are not really nanowire-related patents. To give an example, there are many documents that mention nanowire related terms such as, "this new material also can be used with nanotubes, nanowires and nanocrystals," but the patent is not really related to nanowire patents. This group is very difficult to eliminate from the patent data as it contains cases categorized under nanotechnology related categories, so the only way of eliminating these patents is to examine patents individually. The third group are those patents that include "nano" terms but are not nanotechnology-related patents, such as nanosecond or the iPod nano. Patents in this group are easy to eliminate using the patent collection method used in this study as it consists of patent codes with lexical queries where 
nanowire and nanotechnology related terms are used. The last patent type comprises those patents that are classified under the nanotechnology category such as B82 or 977, but are not nanotechnology-related patents. There are many micro structural related patents under these categories and the main problem with them is that they are not really nanotechnology-related patents, given the requirements and the definition of the nanotechnology field, However, this issue is improving as the B81 (micro structural technology) classification is now being used more carefully and there are assigned teams that work on this issue. If only a list of "nano" terms is used to collect required patents, there is a big possibility that unrelated patents will be collected. Moreover, if one attempts to exclude unnecessary patents by utilising such terms as "-nanosecond*", there is a possibility that required patents also will be excluded, as there is a significant number of patent documents which mention nanotechnology related terms and nanoseconds. It can be argued that there is a possibility of having non-nanowire related patents or missing nanowire related patents in the collected data due to the issues stated above. However, this patent collection method is an effective method in terms of having higher reliability and validity of patent data when compared to other patent collection methods. Huang et al. [19] categorised lexical and patent classification queries by analysing related methodological studies. Porter et al. [55], Mogoutov and Kahane [56] and other similar studies have used lexical queries to gather all patents with nano terms but excluding those patents that have non-related nano terms such as 'nanosecond'. Given the limitations and drawbacks of the above approaches, our method uses a combination of the two, as we use both patent classifications and lexical queries. The reason why both approaches are utilised is because as is mentioned in Scheu et al.'s [57] study, using only patent codes has a weakness in that unrelated patents appear in the patent data due to their wrong classification. Also, using only lexical queries as suggested by Porter [55] resulted in with almost 140.000 patents among which were found many unrelated patents after reviewing samples from the collected 
data. Moreover, even if the data were optimized further, results would not be noticeably

414 different given the type of analysis being followed.

415 As a result, 4484 nanowire patents were analysed with the data covering all the granted and applied patents until March 2012. The obtained results were imported into the Thomson Data

417 Analyser (TDA) and, to validate the results further, duplicate results were eliminated and

418 variations of company, inventor, institute, and university names were unified where they appeared as separate patent assignees. After the dataset was prepared, various functions were utilized using the same tool, Thomson Data Analyser, to generate the required analysis.

There are many other relationships that can be captured and visualized with TDA software. TDA software allows the analysis of patent data and their visualization in many ways, such as mapping, clustering and citation networks. TDA software was used to analyse the collaboration level of organisations in terms of patenting activity, the linkages of organisations within/outside their establishment in whichever country they operated, their collaboration with other actors within the nanotechnology innovation system (universities, institutes and corporations) and the technology diffusion process following the linkages between various academic and non-academic organisations.

\section{Results: The Case of Nanowire}

431 Nanowire is one of the most mature nanostructures that are available today and so an analysis

432 of the patents in this field is significant as there are more patent applications for nanowires compared to many other nanotechnology-related fields [58]. Nanowires (also known as quantum wires) are nanostructures less than ten nanometres long [58]. Nanowires consist of two quantum confined directions when compared to other low dimensional nanostructures 
437 (i.e. $\mathrm{Pt}$ ), semiconducting (i.e. $\mathrm{Si}$ ), and insulating (i.e. $\mathrm{SiO} 2$ ) fields, which means that they

438 have a large variety of applications in different industries [58]. For this study, 4484 nanowire

439 patents were analysed with the data covering all the granted and applied-for patents until

440 March 2012. Patent documents were organized according to their priority years (priority

441 dates) as there are two different dates for a patent document; when it is applied for and when

442 it is granted.

443 There are many possible future applications for nanowires. It is possible that silicon 444 nanowires will provide the next architecture for transistor designs [58]. Nanowire transistors can be at least four times faster than traditional silicon devices and could result in highperformance, low-cost, flexible and miniaturized electronic circuitry for many products and 447 applications [58]. Silicon nanowires will be designed to contour transistor channels, surrounded on all sides by a wrap-around silicon oxide, high-K metal gate [59]. These new nanowire transistors will have different characteristics to the best FinFET transistors [59]. FinFET transistors have a three-dimensional gate (FinFET/Tri-Gate) while nanowires have a cylindrical shape so the gate can be in multipoint all around the device [60]. Another promising application of nanowires is likely to be in highly sensitive nanosensors for the detection of single molecules [61]. As nanowires are at a very small scale, when molecules make contact with the nanowires, they will generate a measurable change in the current passing through the nanowires [61]. There are many possible applications for nanowires in nanosensors, one important one being the detection of cancer proteins. This would allow cancer tests to be more accurate in an inexpensive manner [61].

458 Patenting activity for nanowire technology started in 1994, since when there have been 8420 inventors, 1619 organisations and 32 countries involved in nanotechnology patenting activity. 
records was 731 in 2009.. It appears that, there has been a rapid increase in the number of nanowire patents starting from 1999 to 2010 .

$$
\text { [Insert Figure } 3 \text { here] }
$$

\subsection{Nanowire Patents - International Focus}

This section will look at nanowire patenting activity in two separate sub-sections. Firstly, different countries patenting activities are presented in terms of leading and emerging regions to see the general trend. Secondly, linkages between countries are analysed to see how international collaboration occurs in the nanowire field.

\subsubsection{International Involvement in Nanowire Patenting Activity}

Table 2 presents the top countries with regard to patents but the order of leading countries is different for nanowire technology as compared to the whole nanotechnology field. At present, when considering the total number of nanotechnology related patents, consisting of 49544 nanotechnology patent dataset, the US is the top country, while Korea and China are below Japan, but in the case of nanowire patents, Korea and China have now overtaken Japan. It is remarkable that the number of nanowire patents granted to or applied for by Korean organisations is nearly twice the number of those granted to or applied for by Japanese ones, even though Korea became involved in nanowire technology 3 years after Japan did (please see Table 3). Also, another Asian player, Taiwan, has emerged as a key player in nanowire technology. As shown in Figure 4, China and Korea are catching up with the US, while Japan continues to grow in the nanowire field. Other countries have shown quite a slow increase in their numbers of patents in this field. 


\subsubsection{International Linkages in Nanowire Patenting Activity}

491 This section examines the linkages between countries in terms of organisational 492 collaborations and involvement in different regions. The TDA software performs multidimensional statistical analysis to identify clusters and relationships among these nodes. The size of a node represents the number of documents that belongs to it, while its centrality represents how often that particular node occurs with other nodes. As shown below in Figure 5, the US appears at the centre of linkages and all presented nanowire patenting regions are linked to the US, so it is clear that highest number of nanowire patents are filed in the US. The closeness of nodes and the thickness of lines are calculated on the basis of the significance level between each node, which in turn is calculated on the basis of how many of those documents belong to the node and how many of those documents are shared with the linked node. For example, if node A has twenty documents and ten of those are shared with node $\mathrm{B}$, and five of those are shared with node $\mathrm{C}$, nodes $\mathrm{A}$ and $\mathrm{B}$ would have thicker line between them whereas nodes $\mathrm{A}$ and $\mathrm{C}$ would have a weaker line. The closeness of these nodes is based on the ratio of shared documents between nodes. If node B has ten documents in total and they are all shared with node A then these two nodes would be very close to each other. Considering the significance level, the linkages between US-KR, US-TW and US-SG appear to be the highest in comparison to other linkages. This is calculated according to the number of total patents and number of shared patents that are granted/applied for within those regions. The high significance between US-KR is mainly due to patenting activity of 
510 Samsung in both regions. Moreover, Samsung's patenting activity in this field has resulted in

511 KR being part of the second highest number of linkages in this field. The distance between

512 ego points designates the closeness of the relationship between regions, and so US and JP

513 appear to have a strong linkage as well. Even though $\mathrm{CN}$ is one of the key regions in terms of

514 number of patents, this region does not appear to have a high number of linkages and it

515 appears isolated compared to other leading countries in this field. Referring back to the

516 linkage mechanism that was introduced in the literature review section, this figure illustrates

517 the fact that the current structure of international linkages still very much has the US at the

518 centre. However, it is moving towards a decentral-linkage network structure as KR gains

519 significant positions and an increasing number of linkages with other countries.

[Insert Figure 5 here]

521

522

\subsection{Collaborative Networks and Clusters in the Nanowire Field}

523

524

525

526

527

528

529

530

531

532

533

\subsubsection{Organisational Involvement in Nanowire Patenting Activity}

As shown in Table 4, the leading organisations in the nanowire field are Samsung, HewlettPackard and IBM. All the top electronics companies except Hewlett-Packard became involved in nanowire patenting activity after the millennium. IBM has been granted $54 \%$ of their nanowire patents within the last three years, which indicates their growing interest in this field, probably as a result of its applicability in electronics. This table proves the fact that the key applicability of nanowires is in the electronics industry, as the main patent holders in the field are the top players in that particular industry. The dominant countries for this technology with regard to top organisations appear to be the US and Korea. Examining Samsung's progress, it can be seen that their involvement in nanotechnology started with their focusing on nanowire technology. Even though Samsung are a recent player in 
nanotechnology compared to other companies such as IBM, $17 \%$ of its nanowire patents have been granted within the last 3 years.

536 Table 4 also shows a notable involvement of academic institutions in nanowire technology.

537 For example, the University of California appears to be a leading academic player, which strengthens US dominance even further. In addition, the Korean institutions, the University of Seoul and University of Korea, play a vital role in the technology diffusion process. These academic institutions' involvement may positively affect the commercialisation process in view of their high number of granted patents and their role within technology transfer activity networks.

Another dominant player, Nanosys, was only founded in 2001 and their first involvement with the nanowire field started in 2002. In the last three years, they have not performed well, as they have only been granted $4 \%$ of their overall nanowire patents in this time, but they still play a key role within this sub-domain. The French government-funded technological research organisation, CEA, appears to be the second highest organisation in terms of progress, considering that $43 \%$ of their nanowire patent documents have been granted within the last three years. However, it is notable that even though CEA has a strong dominance in the nanowire field, there is no French corporation within the top players. This may be due to poor collaboration between academic and non-academic organisations in France.

556 In the case of nanowires, the strongest link appears to be between Hon Hai Precision

557 (Foxconn) and Qinghua University (Tsinghua University) (see Figure 6). These two 
organisations share 20 patent documents within the realm of nanowire technology. The second highest number in patent collaboration is between two South Korean players, Samsung and Seoul National University, with their 14 shared nanowire patent documents. Seoul National University (SNU) is one of the leading players in graphene as well, and Samsung and SNU collaborate in various nanotechnology fields. The third highest degree of collaboration is between Samsung and Sungkyunkwan University, with 12 shared patents within the nanowire field.

By looking at the general picture for nanowire technology, the strongest cluster occurs in South Korea (cluster 2). South Korea appears to have a highly centralized network around Samsung and there are some international linkages with other networks. It is to be expected that US players (cluster 1) should be in the centre of nanowire patent activity collaboration as the US has the highest number of nanowire patents, but South Korea has a greater degree of collaborative involvement. The US cluster appears to be decentralized and this type of cluster has better characteristics in terms of its stability and efficiency. Another interesting result that can be gathered from Figure 6 is that even though there are high numbers of patents in Japan, the Japanese nanotechnology cluster (cluster 4) does not look very effective when the number of collaborative nanowire patents is considered. With regard to US-based collaborations, universities and academic institutions appear to have the strongest relationships, such as that between Harvard University, State University of New York and Massachusetts Institute of Technology.

China does not appear to have a cluster but the linkages between Chinese organisations are very significant (cluster 5). In fact, the strongest bond is found between Tsinghua University and Foxconn. However, this is due to their special collaboration terms by which both organisations share all of their nanotechnology-related patents. Moreover, their linkage is a mono-linkage, as it is presented in the proposed model and it appears to be an effective model 
considering the number of shared patents produced. This kind of structure may be an effective model due to two factors. Firstly, it is a linkage between an academic and industrial player so there is great mutual interest in each other's activities and involvement. Secondly, the size of the organisations is significantly large and it is very balanced in respect to their own academic and industrial activity. This is very important for the nanowire field given the fact that required investment is high in respect to the related industries such as the semiconductor industry and it requires scientists from very diverse scientific departments such as material sciences, electronics, and chemistry.

Cross-country collaboration can also be found. The strongest collaboration between US and Korea is that between Hewlett-Packard and two key Korean players, namely Samsung and Sungkyunkwan University. Another strong international collaboration appears between the US and France as was seen when the nanotechnology field was analysed as a whole (cluster 3). In the case of nanowire, the strongest linkage appears to be between CNRS and the California Institute of Technology. Some large organisations are not involved in any collaboration in nanowire patenting activity, such as IBM, Sony and Toshiba. IBM owns 100 nanowire patents and none of these patents is the result of any type of collaboration.

Looking at the general structure of nanowire technology networks and clusters, it can be claimed that the structure of innovation systems may begin with a key collaboration between two or more organisations which agree to form the bidirectional linkage or the first narrowscoped cluster as in the China case. This new formation enlarges and establishes the centralized cluster due to the presence of a dominant player in the system such as Samsung. After the development of centralised clusters, the structure evolves to a decentralized cluster model as in the US case. The next stage is the international connection of organisations that takes place as the cluster moves to the stage where there is a network established. For this case, Samsung is a great example when one sees how they created their network of national 
and international linkages. It is also interesting to see the progress of the Korean innovation system in the nanowire case as a marketing-oriented network moves towards being a complete innovation network.

[Insert Figure 6 here]

\subsubsection{Visualisation of an Organisational Cluster - The Case of Samsung}

In the previous section, key players are identified in terms of linkages with other organisations. Considering the number of patents and linkages in this field, the cluster containing Samsung was chosen for analysis to examine the details of a collaboration mechanism. Following this type of analysis it is possible to see the internal linkages between their collaborative scientists as well.

Figure 7 shows Samsung's nanowire patenting cluster in terms of co-ownership of patent documents. This cluster consists of a central-linkage mechanism and it is highly reliant on the patenting activity of Samsung. It appears that 260 of Samsung's nanowire related patent documents are not co-owned and this shows that Samsung relies on in-house R\&D, as overall that would equate to over $80 \%$ of total patents being generated without collaboration. Depending on Samsung's legal agreements, it may also be the case that Samsung appears as the only holder of those patents even though some of those are the result of collaborations. However, given the significant ratio of co-owned patents to single-owned patents $(0.17)$, this is a noteworthy indication of Samsung's successful internal collaboration for the generation of nanowire-related patents.

[Insert Figure 7 here]

To evaluate possible effects of a central network, Figure 8 is presented to illustrate how South Korea's linkage mechanism would vary if Samsung's significant input did not exist. It is, of 
course, not possible to claim what the linkages would be if Samsung had never existed;

633

634

635

636

637

638

639

640

641

642

643

644

645

646

647

648

649

650

651

652

653

654

655 however, this section examines the potential effects on a central network if the dominant player were missing. Taking into account Samsung's current position, such a dramatic change is not expected; however, this may be the case in a central-linkage mechanism if an organisation such as Samsung minimizes or suspends their investment within a particular field. In this case, it appears that South Korea's central-linkage mechanism would change into a structure of mono-linkages in the case of Samsung's absence. The collaboration structure would completely change and the number of linkages would decrease in a noteworthy fashion. One of the most drastic changes in the Figure is that the linkages between academia and industry would almost disappear and be replaced by linkages between academic institutions. This can be a very dangerous outcome of such a change in a network with regard to the efficiency of technology transfer and commercialisation of nanowirerelated technology. Overall, South Korea's current network appears efficient in terms of granted/applied nanowire patents but its structural risk factors should be avoided in similar networks that have a central-linkage structure. As a result, it can be stated that outside of Samsung most linkages are academic, which indicates that other Korean corporations are not as driven to collaboration with academia or there are possible barriers inhibiting these kinds of collaborations in nanowire technology.

[Insert Figure 8 here]

Table 5 below shows the top three collaborators with Samsung in descending order of number of patents under co-ownership. All of Samsung's collaborations appear to have been with South Korean academic players. If the percentage of shared patent records is examined for the last three year period, the increasing importance of collaborations between academic 
656

657

658

659

660

661

662

663

664

665

666

\section{Discussions and Conclusions}

668

669

670

671

672

673

674

675

676

677

678 collaboration mechanism. nanowire patent documents granted.

and corporate organisations can be seen, as at least $10 \%$ of collaborations happened in this period with each actor. Moreover, it is possible to see if the collaboration mechanism is a continuous process, since it is possible to see the time period when these organisations are collaborating. If a visual network figure was used, it would only be possible to see the number or types of linkages, but it would not be possible to see which are active or passive.

This table also allows the reader to see the key inventors that play important roles in terms of collaborations between these organisations. Accordingly, this type of study can be used as the basis for a qualitative study of these key inventors to gather determinants about the

[Insert Table 5 here]

In this article, nanowire patent documents were carefully analysed with four foci, which are international, organisational, technological and institutional. In addition, this paper explored different models within innovation system theory and various network and cluster models were examined to form the theoretical basis of the study.

The international profile of nanowire technology provided valuable information, such as key regions, with regard to the number of nanowire patents. This research has also presented country-based key technology domains and dominant players within those countries. An interesting outcome was to see the changing trend of countries' involvement in nanowire technology as Asian players in the last year had huge involvement in this area. It appears that South Korea and China are now ahead of Japan and close to the US in terms of the number of 
679 Considering the networks or clusters for nanowire technology, it can be said that these vary 680 greatly from one country to another. It was found that the largest network was Samsung's 681 centralized network in South Korea. This network has international linkages with other 682 countries, for example with organisations based in the US. This is due to the international 683 externalities of multinational companies such as Samsung. On the other side, talking about international externalities, the biggest collaboration was identified as being between the US and France in nanowire patenting activities. There was a high degree of co-ownership by French and US organisations both in the academic and private spheres. However, it was found that the main focus of these relationships was within the electronics sector. This is of course due to the application of nanowire technology to semiconductors, batteries and display technologies. From the point of view of the proposed network model, with five different classifications of mono-linkage, oligo-linkage, central-linkage, decentral-linkage and distributed linkage, the general structure of nanowire networks was found to be somewhere between centralised and decentralised and very far from being a distributed network structure. That means the network relies greatly on organisations such as Samsung, which dominates the Korean centralised network. It would be expected that the US would have the highest number of linkages considering the fact that it is at the centre of nanowire patenting activity in the international linkages figures, but looking at patent activities at the organisational level, it can be noted that the US has a national cluster rather than a network and the number of collaborating organisations is lower than in the Korean case. Another surprising fact that can be gathered from this analysis is that the Chinese collaboration mechanism is not very strong in terms of linkages between private and public organisations. The key linkage in the Chinese context is between Tsinghua University and Foxconn, an organisation with its headquarters in Taiwan, but which has most of its production assets in China. In China, the number of collaborative organisations should be increased to move it to 
the stage where there is an innovative cluster to increase the technology diffusion process. This research suggests that the government should take action to bring this about.

With respect to the key actors within the nanowire case, it was found that within the electronics industry, ownership of patents is dominated mostly by large organisations. There are two main reasons why there is considerable heterogeneity in nanowire patenting activity. Firstly, large organisations have the capability to provide the huge investment necessary for R\&D activities, and they are aware of the benefits of nanowire technology in terms of its efficiency and its nature for bringing about incremental innovative characteristics. Secondly, they collaborate with academic organisations such as universities and institutions to benefit from their inventions as well. The second point is not found in every national innovation system, but Korea, the US and Japan appear to have a more effective environment compared to other nations in this case.

To summarise the important implications of this study, the following conclusions are listed:

Asian organisations, especially in South Korea and the Chinese region appear to be having a great impact in the nanowire field.

Considering the linkages between organisations, there is a sector concentration in the electronics industry in terms of patenting activity, especially in central linkage mechanisms. This is due to the large investment of global players and their related interest in nanowire applications such as semiconductors and energy storage-related devices.

In terms of collaboration and innovation models, nanowire technology was found to be in its initial stage where various centralised clusters or networks exist. However, some nations such as South Korea, US and JP are far ahead in terms of number of linkages between academia and industry in the nanowire field. 
727 In relation to this study a path is proposed for innovation systems; that is key participants lead 728 to clusters, and clusters to networks, and networks result in innovation systems. This was the result of examining the progression of various nations and organisations involved in nanowire technology. For example, in the case of mono-linkages in $\mathrm{CN}$, it would be expected that their linkage mechanism would move into an oligo-linkage, a central-linkage or a decentrallinkage and this would lead to a network after the region progresses further.

This paper also illustrates a great example of a central network by using Samsung's cluster. When considering the efficiency of this model, there are many points open to argument, as various scientists have proposed different ideas in terms of large players' involvement in innovation activity. Looking at this type of collaboration mechanism purely based on type of linkages as it is mentioned in the TEN model (Technology Pole, Science Pole and Market Pole) and structure of linkages (mono-linkage, oligo-linkage, central-linkage, decentrallinkage and distributed linkage), if the central ego (Samsung in the KR case) were missing or if their contribution was minimized due to various factors, it would affect the whole network, as some of the mentioned poles would disappear or be minimized to a low number of linkages or technology transfer processes, or the diffusion of technology would be drastically affected as the whole network would need to form into a new model. This was also illustrated by examining the structure of KR with and without Samsung to see the possible differences in the network. It was obvious that the number of linkages would be drastically reduced and linkages between academia and industry would almost completely disappear. Considering the fact that even large players struggle in any type of market (even large automobile players) and given Samsung's crises in 1997, it can be expected any central network will risk losing its structure in crises as compared to a decentral-linkage and distributed linkage structure. nanowire technology. As was mentioned in the findings section, there are some organisations 
and inventors that hold a high number of nanowire patent documents but the question is whether they are highly influential patents in terms of citations, commercial potential and quality. Accordingly, a follow-up study could be conducted on nanowire patent documents to look at this field in terms of quality in comparison with quantity.

\section{Acknowledgement}

The authors gratefully acknowledge the valuable advice of Prof. Fred Phillips, Prof. Philip Shapira and Prof. Scott W. Cunningham. The helpful comments and suggestions of the anonymous reviewers are also gratefully acknowledged. They wish to express their gratitude for the financial support given by Rowland's Foundation and the Aberystwyth University Research Fund. The initial version of this paper was presented at the International Conference on Innovative Methods for Innovation Management and Policy (IM2012), Beijing and thanks are given for suggestions made by those experts who were at the conference.

\section{References}

[1] J.J. Ramsden, What is Nanotechnology? Nanotechnology Percetions. 2005.

[2] I. Rafols, M. Meyer, Diversity and network coherence as indicators of interdisciplinarity: case studies in bionanoscience. Scientometrics, 82 (2) (2010) 263-287.

[3] A.L. Porter, J. Youtie, How interdisciplinary is nanotechnology? Journal of Nanoparticle Research 11 (5) (2009) 1023-1041.

[4] I. Rafols, M. Meyer, How cross-disciplinary is bionanotechnology? Explorations in the specialty of molecular motors. Scientometrics, 70 (3) (2007) 633-650.

[5] N. Islam, K. Miyazaki, Nanotechnology innovation system: Understanding hidden dynamics of nanoscience fusion trajectories, Technological Forecasting and Social Change, 76 (1) (2009) 128-140.

[6] P. Murphy, D. Munshi, P.A. Kurian, A. Lakhtakia, R.V. Bartlett, Nanotechnology, Society, and Environment, In: Ed. David L. Andrews, Gregory D. Scholes, and Gary P. Wiederrecht, Comprehensive Nanoscience and Technology, Academic Press, Amsterdam, 2011 443-476.

[7] V. Mangematin, S. Walsh, The future of nanotechnologies, Technovation, 32 (3-4) (2012) 157160.

[8] T. Rogers-Hayden, N. Pidgeon, Developments in nanotechnology public engagement in the UK: 'upstream' towards sustainability? Journal of Cleaner Production, 16 (8-9) (2008) 1010-1013. 
[9] J. T. Lue, Physical Properties of Nanomaterials, Encyclopedia of Nanoscience and Nanotechnology, American Scientific Publishers. USA. 2007, 10-12.

[10]C. Lee, J. Jeon, Y. Park, Monitoring trends of technological changes based on the dynamic patent lattice: A modified formal concept analysis approach, Technological Forecasting and Social Change, 78 (4) (2011) 690-702.

[11]C. Choi, Y. Park, Monitoring the organic structure of technology based on the patent development paths, Technological Forecasting and Social Change, 76 (6) 2009 754-768.

[12] A.K. Firat, W.L. Woon, S. Madnick, Technological Forecasting - A Review, Massachusetts Institute of Technology, 2008.

[13] T. U. Daim, G. Rueda, H. Martin, P. Gerdsri, Forecasting emerging technologies: Use of bibliometrics and patent analysis, Technological Forecasting and Social Change, 73 (8) (2006) 981-1012.

[14] R.N. Kostoff, D.R. Toothman, H.J. Eberhart, J.A. Humenik, Text mining using database tomography and bibliometrics: A review, Technological Forecasting and Social Change, 68 (3) (2001) Pages 223-253.

[15]I. von Wartburg, T. Teichert, K. Rost, Inventive progress measured by multi-stage patent citation analysis, Research Policy, 34 (10) (2005) 1591-1607.

[16] K. Chang, D. Chen, M. Huang, The relationships between the patent performance and corporation performance, Journal of Informetrics, 6 (1) (2012) 131-139.

[17] K. Miyazaki, N. Islam, Nanotechnology systems of innovation-An analysis of industry and academia research activities, Technovation, 27 (11) (2007) 661-675.

[18]Z. Huang, H. Chen, A. Yip, G. Ng, F. Guo, Z. Chen, M. C. Roco, Longitudinal patent analysis for nanoscale science and engineering : Country, institution and technology field. Journal of Nanoparticle Research. 5 (3-4) (2003) 333-363.

[19] Li, X., Chen, H., Huang, Z., Roco, M. Patent citation network in nanotechnology (1976-2004). Journal of Nanoparticle Research, 9 (3) (2007) 337-352.

[20] A.L. Porter, J. Youtie. Where does nanotechnology belong in the map of science. Nat Nano, Vol. 4, No. 9, Nanotech, Pages 534-536. 2009.

[21]P. Shapira, J. Youtie, L. Kay, National innovation systems and the globalization of nanotechnology innovation. The Journal of Technology Transfer. 36 (6) (2011) 587-604.

[22] J. Schumpeter, The Theory of Economic Development, Cambridge, Mass: Harvard University Press 1934.

[23] W.E. Souder, Improving productivity through technology push, Journal of Product Innovation Management, 6 (4) (1989) 305-306.

[24] A. Brem, K. I. Voigt, Integration of market pull and technology push in the corporate front end and innovation management-Insights from the German software industry, Technovation, 29 (5) (2009) 351-367.

[25]C. Herstatt, C. Lettl, Management of 'technology push' development projects, International Journal of Technology Management, 27 (2/3) (2004) 155-175.

[26] V. Walsh, Invention and innovation in the chemical-industry - demand-pull or discovery-push. Research Policy 13 (4) (1984) 211-234.

[27] S.T. Walsh, B.A. Kirchhoff, S. Newbert, Differentiating market strategies for disruptive technologies, IEEE Transactions on Engineering Management, 49 (4) (2002) 341-351.

[28] S.R. Chidamber, H.B. Kon, A research retrospective of innovation inception and success - the technology-push, demand-pull question. International Journal of Technology Management 9 (1) (1994) 94-112. 
[29]F.M. Scherer, Demand-pull and technological invention - Schmookler revisited. Journal of Industrial Economics 30 (3) (1982) 225-237.

[30] G. Nemet, Demand-pull, technology-push, and government-led incentives for non-incremental technical change, Research Policy, 38 (5) (2009) 700-709.

[31]B. Lundvall, National Systems of Innovation: Towards a Theory of Innovation and Interactive Learning, London, Pinter. 1992.

[32] X. Liu, S. White, Comparing innovation systems: a framework and application to China's transitional context, Research Policy, 30 (7) (2001) 1091-1114

[33] J. Guan, K. Chen, Modeling the relative efficiency of national innovation systems, Research Policy, 41 (1) (2012) 102-115.

[34]D. Doloreux, What we should know about regional systems of innovation, Technology in Society, 24 (3) (2002) 243-263

[35]D. S. Yim, B. Kang, Policy Options for Establishing Effective Subnational Innovation Systems and Technological Capacity-building, Asia-Pacific Trade and Investment Review, 4 (2008) 115137.

[36] S. Roper, J. Du, J. H. Love, Modelling the innovation value chain, Research Policy, 37 (6-7) (2008) 961-977.

[37]C. Freeman, The National System of Innovation in Historical Perspective, Cambridge Journal of Economics, 19 (1995) 5-24.

[38] R. Nelson, National Innovation Systems, Oxford University Press, Oxford, 1993.

[39] P. Cooke, K. Morgan, The Creative Milieu: a Regional Perspective on Innovation, in M. Dodgson, et.al., The Handbook of Industrial Innovation, (1994) 57-89.

[40]P. Cooke, K. Morgan, The Associational Economy: Firms, Regions and Innovation, Oxford University Press, 1998.

[41]F. Malerba, Sectoral Systems of Innovation: Concepts, Issues and Analyses of Six Major Sectors in Europe, 2004.

[42]B. Carlson, R. Stankiewicz, On the nature, function and composition of technological systems, Journal of Evolutionary Economics, (1) (1991) 93-118.

[43] A. Johnson, Functions in innovation system approaches, Department of Industrial Dynamics, Chalmers University of Technology, 1998.

[44] T. Kirat, Y. Lung, Innovation and proximity: territories as loci of collective learning processes. European Urban and Regional Studies, 6 (1) (1999) 27-38.

[45] T. Velden, A. Haque, C. Lagoze, A new approach to analyzing patterns of collaboration in coauthorship networks: mesoscopic analysis and interpretation, Scientometrics, 2010.

[46]C. Ketels, The impact of clusters and networks of firms on EU competitiveness, Harvard Business School, Final Report: Firm networks, 2012.

[47]D. Mercedes, M.E. Porter, S. Stern, Clusters, Convergence, and Economic Performance, Census Working Paper, 2011.

[48] L.M. Camarinha-Matos, H. Afsarmanesh, Collaborative Networks: Value creation in a knowledge society, International Federation for Information Processing IFIP, 207 (2006) 26-40.

[49] OECD, National Innovation System, Paris: OECD, 1997

[50] L. Leydesdorff, M. Meyer, The Triple Helix of university - industry - government relations. Scientometrics, 58 (2) (2003) 191-203.

[51]D.R. Paudyal, K. McDougall, A. Apan, A Regional Collaborative Network to Improve Spatial Information Sharing in Australia, 12th Global Spatial Data Infrastructure Association World Conference, Singapore, 2010. 
[52] A.L. Porter, S.W. Cunningham, Tech mining: exploiting new technologies for competitive advantage. John Wiley and Sons. New Jersey, 2005.

[53] K. M. T. Collins, A. J. Onwuegbuzie, Q. G. Jiao, A mixed methods investigation of mixed methods sampling designs in social and health science research. Journal of Mixed Methods Research, 1 (2007) 267-294.

[54] A. Tashakkori, C. Teddlie, Mixed Methods in Social and Behavioural Research, Sage Publications Inc, 2003.

[55] Porter, A. L., Youtie, J., Shapira, P., Schoeneck, D. J., 2008. Refining search terms for nanotechnology. Journal of Nanoparticle Research. 10(5), 715-728. Springer. Netherlands.

[56] Mogoutov, A., Kahane, B., 2007. Data search strategy for science and technology emergence: A scalable and evolutionary query for nanotechnology tracking. Research Policy, 36, 893-903.

[57] M. Scheu, V. Veefkind, Y. Verbandt, E. Galan, R. Absalom, W. Förster, Mapping nanotechnology patents: The EPO approach. World Patent Information. 28(3), 204-211 2006.

[58] M.S. Dresselhaus, Y.M. Lin, O. Rabin, M.R. Black and G. Dresselhaus, Nanowires, Springer Handbook of Nanotechnology, (2004) 99-145.

[59] M. Shin, Efficient Simulation of Silicon Nanowire Field Effect Transistors and Their Scaling Behaviour. Journal of Applied Physics, 101 (2) (2007).

[60]P. Sangwoo, M. Jose, D. Gilbert, V. M. Matthew, K. Markus, D. Mark, K. Jack, High quality silicon oxynitride transition layer for high- $\mathrm{k} / \mathrm{metal}$ gate transistors. Application number: 11/729,188. Current U.S. Classification: 438/197, 2007.

[61]F. Patolsky, G. Zheng and C. M. Lieber, Nanowire sensors for medicine and the life sciences. Nanomedicine, Vol. 1, No. 1, Pages 51-65, 2006. 